To be published in Optics Letters:

Title: Internal modulation of a Random fiber laser

Authors: Mikel Bravo, Montserrat Fernandez-Vallejo, and Manuel López-Amo

Accepted: 2 April 2013

Posted: 3 April 2013

Doc. ID: 186039

(C) 2013 Optical Society of America. One print or electronic copy may be made for personal use only. Systematic reproduction and distribution, duplication of any material in this paper for a fee or for commercial purposes, or modifications of the content of this paper are prohibited. 


\title{
Internal modulation of a Random fiber laser
}

\author{
M. Bravo ${ }^{1 *}$, M. Fernandez-Vallejo ${ }^{1}$, M. Lopez-Amo ${ }^{1}$. \\ ${ }^{I}$ Departamento Ingeniería Eléctrica y Electrónica, Universidad Pública de Navarra, Campus Arrosadia S/N, Pamplona. \\ 31005, Spain \\ *Corresponding author: mikel.bravo@unavarra.es
}

Received Month X, XXXX; revised Month X, XXXX; accepted Month X, XXXX; posted Month X, XXXX (Doc. ID XXXXX); published Month X, XXXX

\begin{abstract}
A characterization of a modulated random mirror laser has been experimentally carried out. Unlike conventional internally modulated fiber lasers, no distortion of the modulating frequency or self-mode-locking effects were measured. The behavior of the laser using pulsed and analog modulation up to $12 \mathrm{GHz}$ is shown. (C) 2013 Optical Society of America OCIS Codes: $060.3510,060.4080$
\end{abstract}

For a long time, optical scattering was considered detrimental to conventional lasers because such scattering removes photons from the lasing modes. Over recent years, however, random lasers based on Rayleigh scattering feedback and amplified through Raman Effect have been the subject of intense theoretical and experimental study. Random lasers are characterized by open-cavities or mirror-less-cavities which means that, unlike usual lasers, their principle of operation rely on multiple scattering events. Rayleigh scattering in the fiber core is extremely weak, but its effect is accumulated and amplified over a long distance, thus laser condition is fulfilled. Random fiber lasers [1-7] have attracted a lot of attention because of their advantages such as stability and special spectral properties based on the fact that they do not need "real" mirrors to create the cavity. They also offer high lasing efficiency and they are able to reach long distances. In particular, ultra-long fiber lasers have become an attractive solution for transmission communications links because longer repeater spacing entails lower cost. This kind of lasers, usually, uses the simplest linear configurations and with the aid of distributed Raman gain along the fiber reaches long distances. Although the length of the first approaches was less than $100 \mathrm{~km}$, the deep research on this area has contributed to expand this length considerably [1-7].

Fiber lasers can offer not only continuous-wave (CW) operation but also they can be internally modulated in order to generate pulses or RF signals. These modulated lasers have a wide range of appealing applications such as sources in communication, for Optical Time Domain Reflectometry (OTDR), in spectroscopy; as sources for interrogating fiber optic sensors; or as tunable seed pulses for lasers in medical applications, and so on.

Each application is determined by the wavelength operation and bandwidth or repetition rate in pulsed lasers. Generally speaking, modulated fiber lasers $\left(\mathrm{Q}^{-}\right.$ switched [8] or mode-locked ones [9]) can be actively or passively modulated depending on if an amplitude or phase modulator is included or not in the cavity. The passive technique is the preferred one when very short light pulses, in the order of femtoseconds, are required. On the contrary, actively modulated fiber lasers offer high repetition rates, or frequency modulation, and good extinction ratios. The advantage of the active $\mathrm{Q}$ switch is the easy control of the pulse repetition rate, and consequently, the pulse width, whereas the disadvantage is the requirement of an optical modulator.

In this letter, a random fiber laser cavity has been modulated through an intensity modulator. Unlike conventional mode-locked lasers where the length of the laser resonator is an essential parameter because of the fact that it determines the repetition rate [10-12]; in the proposed modulated random fiber laser the repetition rate can be customized. To the best of the authors' knowledge, this is the first demonstration of an internally modulated random laser.

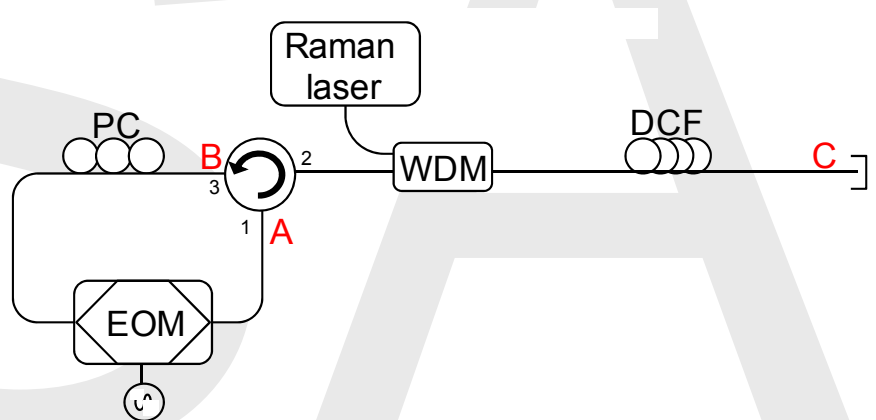

Fig. 1. Schematic setup for the proposed modulated laser system. Point C is adapted. PC: Polarization controller. EOM: Electrooptical modulator. WDM: Wavelength division multiplexer. DCF: Dispersion compensating fiber.

Figure 1 depicts the schematic setup of the internally modulated random fiber laser amplified by the Raman effect using a $1445 \mathrm{~nm}$ pump laser. This laser is based on a linear cavity formed of two different mirrors: the first one (right side), a distributed mirror created by using a $\sim 2.5 \mathrm{~km}$ DCF (Dispersion Compensated Fiber) reel and the second one (left side), a loop mirror by connecting the inputs/outputs of a circulator as shown in Fig. 1.

The operation mode of the system is as follows. The loop mirror structure receives the reflected light from the distributed mirror by port 2 . Once light is inside the mirror (between port 3 and 1), this light is modulated by a LiNb03 Mach-Zehnder EOM (Electo-Optical Modulator) with an extinction ration of $\sim 20 \mathrm{~dB}$. The signal selected to modulate the structure is a pulsed shape which causes the on/off switching. Once light is modulated, it is launched through port 2 towards the distributed mirror. It is 
important to highlight the role of the loop mirror structure because of the fact that the reflected signal is always modulated. It could be called "modulated mirror structure", specifically; the modulated mirror modulates the backscattered contribution generated by the distributed mirror. Due to the particular features of the random cavity, its feed-back action does not correspond to a fixed length; and the cavity adapts without distortion to each selected frequency of modulation. It means that the laser cavity does filter periodically in frequency the amplified signal, as in Fabry-Perot lasers. Thus, the contribution of each point of the DCF fiber which arrives to the modulator in phase will be re-coupled into the cavity and, when the gain compensates the losses, creates a positive feedback achieving the laser condition. The entire backscattered signal created in the cavity is accumulated in point $B$. point A. The spectrum has been also checked in points B and $\mathrm{C}$ obtaining similar results in order to confirm the laser operation along the whole cavity. The output spectra of points A and B were measured using a 90:10 low insertion loss coupler and the measurement at $\mathrm{C}$ was taken directly from the fiber tip. It is important to remark that modulating internally the laser, the cavity loss is initially kept at a high level until the pumped gain medium has stored a certain amount of energy. The cavity loss is then quickly reduced to a small value, which allows the intense stimulated laser radiation to establish quickly in the cavity [6].

Thus the modulated signal obtained in A is different from the obtained by modulating directly the output signal of a CW random mirror laser. In addition, the measured extinction ratio of the modulated random laser is $4 \mathrm{~dB}$ higher than the extinction ratio of the EOM.

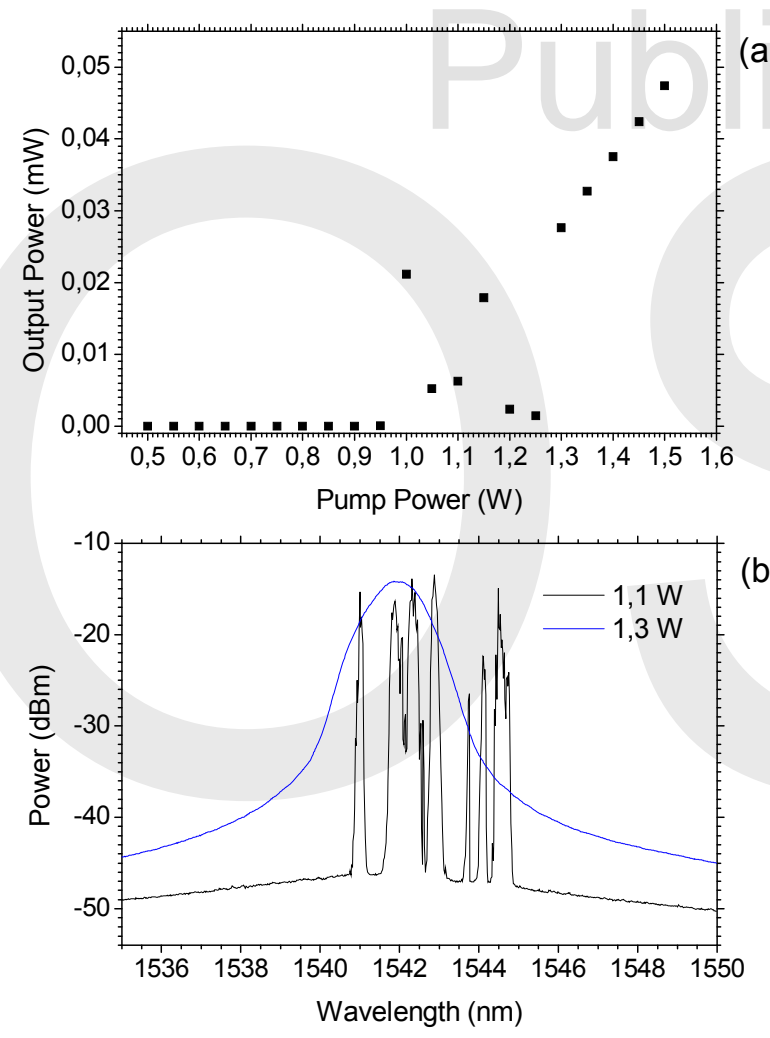

Fig. 2. (a) Output power evolution vs. pump power variation. (b) Output spectrum measurements for three different pump power.

The output power evolution versus the Raman pump power was measured in order to demonstrate the lasing behaviour of the structure (Figure 2 (a)). In the figure, two different zones are noticed. The first one corresponds to an unstable behaviour (from 1 to $1.3 \mathrm{~W}$ ) before the stable lasing behaviour is achieved. In Figure 2(b) the output spectra corresponding to both zones are depicted with a resolution of $0.1 \mathrm{~nm}$. The black trace refers to the aforementioned unstable state where different hopping lasing lines are noticeable; and the blue one belongs to the broadband stable operation regime of the proposed modulated random laser. The measurements corresponding to Figures 2 (a) and (b) have been taken in

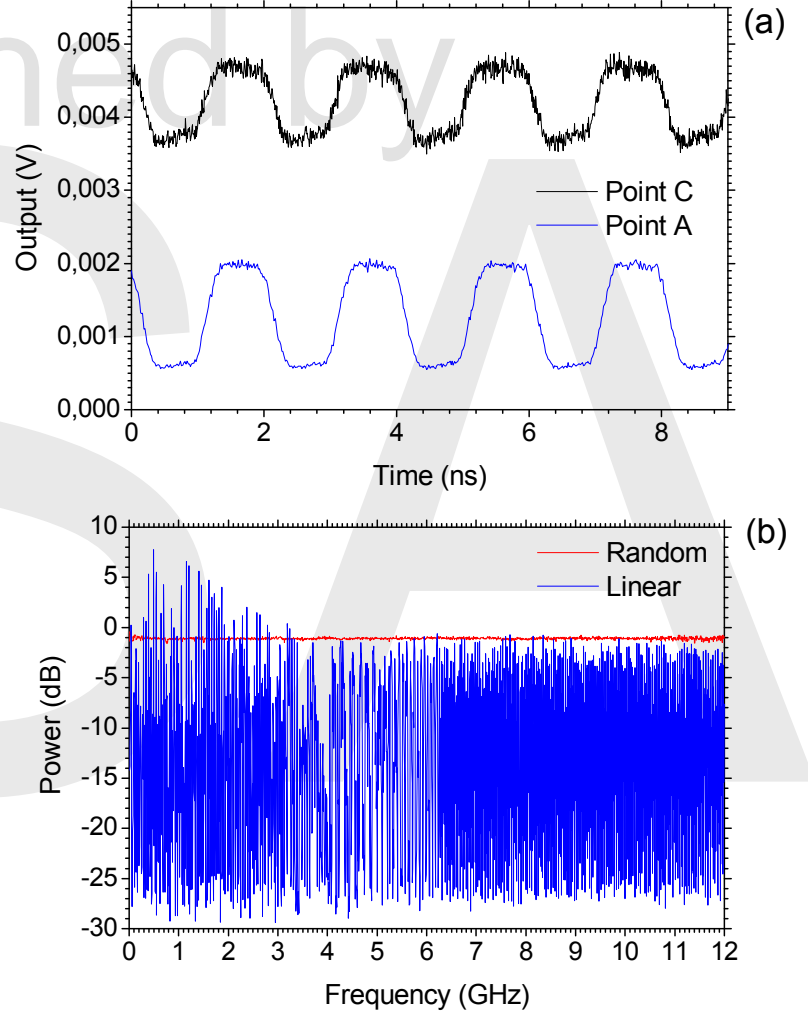

Fig. 3. (a) Time domain laser behaviour in $\mathrm{A}$ and $\mathrm{C}$ when $15 \mathrm{MHz}$ square signal modulates the system. (b) Frequency response of the proposed random cavity in comparison with a linear cavity.

Figure 3 (a) displays a pulsed output signal of the random laser, measured simultaneously at points $\mathrm{A}$ and $\mathrm{C}$ when a $500 \mathrm{MHz}$ square signal is applied to the EOM because it is important to check the behavior of the signal in every point. Although the signal in both points is rather similar, signal in point $\mathrm{C}$ offers much lower contrast than signal in point $\mathrm{A}$. The signal in point $\mathrm{C}$ is noisier because it travels through the DCF along with the Raman pump; the signal is not only amplified by the Raman Effect but also it is affected by additional undesired effects such as the double backscattering which modifies the signal in point C. Furthermore, point A offers a free-noise signal 
due to the fact that the electro-optical modulator removes all the extra noise. This figure demonstrates how the proposed system is able to modulate the laser in a pulsed regime. Because of the frequency limitations of our modulating devices for square signals, no measurements over $1 \mathrm{GHz}$ were carried out. In fact, in figure 3 (a), low frequency filtering effects make smoother the signal shape. A higher frequency operation, nevertheless, is expected with the suitable equipment.

We have also characterized the laser behaviour for high frequency analog modulation.

Figure 3 (b) compares the frequency behaviour of the proposed random mirror laser and a conventional linear cavity laser. A network analyzer was used to characterize both systems from $300 \mathrm{kHz}$ to $12 \mathrm{GHz}$ using a frequency resolution of $200 \mathrm{kHz}$. Both cavities have the same length and topology to compare the lasers behaviour. The random mirror laser has been transformed into a linear cavity laser introducing a FBG (centre wavelength: 1549.6 $\mathrm{nm}$, bandwidth: $\sim 0.25 \mathrm{~nm}$, reflectivity: $\sim 97 \%$ ) at $\mathrm{C}$ to reflect the output power. Thus, the reflected signal overrides the effect of the distributed mirror, being the new cavity length the $\sim 2.5 \mathrm{~km}$ of the DSF. It is remarkable the flat behaviour of the random laser independently of the frequency in comparison to the periodic spectral response of the linear cavity. This frequency dependence is evident in the blue plot from Figure 3 (b) which shows the high number of frequency minima present in the linear cavity.

In conclusion, operation of a random mirror modulated laser has been demonstrated. To the authors' knowledge, this is the first demonstration of this kind of modulated lasers. It has been also demonstrated its lasing operation in different points of the cavity. Unlike conventional modulated lasers where the length of the laser resonator distorts the analog internal modulation or determines the repetition rate of generated pulses; in the proposed modulated random fiber laser the modulation signal is not distorted. This first approach could be enhanced using more suitable equipment. The application of these modulated lasers is appealing for remote measurements if the DCF fiber is replaced by tens even hundreds kilometers of single mode fiber and for time division fiber optic sensors multiplexing systems.

\section{Acknowledgments}

This work was supported by the Spanish Government project TEC2010-20224-C02-01.

\section{References}

1. J. D. Ania-Castañon, T. J. Ellingham, R. Ibbotson, X. Chen, L. Zhang, and S. K. Turitsyn, Phys. Rev. Lett., 96, 2, (2006).

2. S. K. Turitsyn, J. D. Ania-Castañón, S. A. Babin, V. Karalekas, P. Harper, D. Churkin, S. I. Kablukov, A. E. El-Taher, E.V. Podivilov, and V. K. Mezentsev, Phys. Rev. Lett., 103, 13, (2009).

3. S. K. Turitsyn, S. A. Babin, A. E. El-Taher, P. Harper, D. V. Churkin, S. I. Kablukov, J. D. Ania-Castañon, V. Karalekas and E. V. Podivilov, Nature Photonics, 4, (2010).

4. A. E. El-Taher, P. Harper, S. A. Babin, D. V. Churkin, E. V. Podivilov, J. D. Ania-Castanon, and S. K. Turitsyn, Optics Letters, 36, 2, (2011).

5. I. D. Vatnik, D. V. Churkin, and S. A. Babin, Optics Express 20, 27, (2012).

6. H. Martins, M. B. Marques, and O. Frazao, Optics Express 19, 19, (2012).

7. A. M. R. Pinto, O. Frazão, J. L. Santos, and M. Lopez-Amo, Journal of Lightwave Technology, 29, 10, (2011).

8. Y. Wang, C-Q. Xu, Progress in Quantum Electronics, 31, 3-5, (2007)

9. F. K. Fatemi and J. W. Lou,, Optics letters, 29, 20, (2004)

10. B. Vizoso, M. C. Vázquez, R. Civera, M. López-Amo, M. A. Muriel, Journal of Lightwave Technology, 12, 2 (1994).

11. C.E.S. Castellani, E.J.R. Kelleher, Z. Luo, K. Wu, C. Ouyang, P.P. Shum, Z. Shen, S.V. Popov, and J.R. Taylor Laser Phys. Lett. 9, 3, (2012)

12. B. Dong, J. Hao, J. Hu, C. Liaw, "Short linear-cavity Q-switched fiber laser with a compact short carbon nanotube based saturable absorber" Optical Fiber Technology 17, 2, (2011) 
1. J. D. Ania-Castañon, T. J. Ellingham, R. Ibbotson, X. Chen, L. Zhang, and S. K. Turitsyn, "Ultralong Raman fiber lasers as virtually lossless optical media”, Phys. Rev. Lett., 96, 2, (2006).

2. S. K. Turitsyn, J. D. Ania-Castañón, S. A. Babin, V. Karalekas, P. Harper, D. Churkin, S. I. Kablukov, A. E. El-Taher, E.V. Podivilov, and V. K. Mezentsev, "270-km ultralong Raman fiber laser", Phys. Rev. Lett., 103, 13, (2009).

3. S. K. Turitsyn, S. A. Babin, A. E. El-Taher, P. Harper, D. V. Churkin, S. I. Kablukov, J. D. Ania-Castañon, V. Karalekas and E. V. Podivilov, "Random distributed feedback fibre laser", Nature Photonics, 4, (2010).

4. E. El-Taher, P. Harper, S. A. Babin, D. V. Churkin, E. V. Podivilov, J. D. Ania-Castanon, and S. K. Turitsyn, "Effect of Rayleighscattering distributed feedback on multiwavelength Raman fiber laser generation", Optics Letters, 36, 2, (2011).

5. D. Vatnik, D. V. Churkin, and S. A. Babin, "Power optimization of random distributed feedback fiber lasers", Optics Express 20, 27, (2012).

6. H. Martins, M. B. Marques, and O. Frazão, "300 km-ultralong Raman fiber lasers using a distributed mirror for sensing applications", Optics Express 19, 19, (2012).

7. M. R. Pinto, O. Frazão, J. L. Santos, and M. Lopez-Amo, "Multiwavelength Raman Fiber Lasers Using Hi-Bi Photonic Crystal Fiber Loop Mirrors Combined With Random Cavities", Journal of Lightwave Technology, 29, 10, (2011).

8. Y. Wang, C-Q. Xu, "Actively Q-switched fiber lasers: Switching dynamics and nonlinear processes" Progress in Quantum Electronics, 31, 3-5, (2007)

9. F. K. Fatemi and J. W. Lou,, "Wideband frequency modulation of a mode-locked fiber laser Optics letters", 29, 20 (2004)

10. B. Vizoso, M. C. Vázquez, R. Civera, M. López-Amo, M. A. Muriel, "Amplified fiber-optic delay lines "Journal of Lightwave Technology, 12, 2 (1994).

11. C.E.S. Castellani, E.J.R. Kelleher, Z. Luo, K. Wu, C. Ouyang, P.P. Shum, Z. Shen, S.V. Popov, and J.R. Taylor "Harmonic and single pulse operation of a Raman laser using grapheme" Laser Phys. Lett. 9, 3, (2012)

12. B. Dong, J. Hao, J. Hu, C. Liaw, "Short linear-cavity Q-switched fiber laser with a compact short carbon nanotube based saturable absorber" Optical Fiber Technology 17, 2, (2011) 